\title{
Karyotype characterization, constitutive heterochromatin and nucleolus organizer regions of Paranaita opima (Coleoptera, Chrysomelidae, Alticinae)
}

\author{
Mara Cristina de Almeida ${ }^{1}$, Carlos Campaner $^{2}$ and Doralice Maria Cella ${ }^{3}$ \\ ${ }^{1}$ Departamento de Biologia Estrutural, Molecular e Genética, Setor de Ciências Biológicas e da Saúde, \\ Universidade Estadual de Ponta Grossa, Ponta Grossa, PR, Brazil. \\ ${ }^{2}$ Museu de Zoologia da Universidade de São Paulo, São Paulo, SP, Brazil. \\ ${ }^{3}$ Universidade Estadual Paulista, UNESP, Instituto de Biociências, Departamento de Biologia, Rio Claro, \\ SP, Brazil.
}

\begin{abstract}
Species of the subtribe Oedionychina not only have a highly uniform diploid number of $2 n=22(20+X+y)$ but have the karyotypic peculiarity of possessing extremely large sex chromosomes. We analyzed Paranaita opima embryos and gonadal cells to determine their diploid number, chromosomal morphology, type of sex determination system, constitutive heterochromatin pattern and which chromosomes bear nucleolus organizer regions (NORs). The diploid number of $P$. opima was $2 \mathrm{n}=22(20+\mathrm{XY} / \mathrm{XX})$ with all chromosomes being metacentric. Chromosome pair 6 showed an interstitial secondary constriction on the short arm. The C-banding technique revealed centromeric constitutive heterochromatin in all chromosomes, which, in pair 6, extended up to the secondary constriction of the short arm, additional C-bands also being present on the $\mathrm{Y}$ chromosome. Silver nitrate nucleolar organizer region (Ag-NOR) staining showed NORs on the secondary constriction of pair 6 . Fluorochrome analysis with chromomycin $\mathrm{A}_{3}\left(\mathrm{CMA}_{3}\right)$, 4'-6-diamidino-2-phenylindole (DAPI) and the distamycin A (DA) counterstain showed that the short arm of chromosome pair 6 exhibited a GC-rich block extending from the proximal to the median region, including part of the secondary constriction. The same techniques also showed AT-rich blocks at the centromeric region of all chromosomes and at the terminal region of the short arm of pair 6 . The basic karyotype characteristics and $\mathrm{C}$ band pattern of $P$. opima are similar to those described for other species in the subtribe Oedionychina. The pattern of autosomal NORs observed in P. opima corresponds to that registered in the majority of the Chrysomelidae species.
\end{abstract}

Key words: C bands, chromosome, embryos, fluorochromes, NOR.

Received: July 4, 2005; Accepted: December 12, 2005.

\section{Introduction}

The tribe Oedionychini (Alticinae) includes two subtribes, Disonychina and Oedionychina. Cytogenetic studies performed on 20 Disonychina species have shown that this group possesses high inter- and intraspecific variability in relation to the diploid number and type of sex determination system. An idea of the extent of such variation is given by the karyotypic variability described in the Disonycha species D. spilotracheta $\left(2 \mathrm{n}=29\left(13 \mathrm{II}+\mathrm{X}_{1} \mathrm{y}+\mathrm{X}_{2}\right)\right.$, Virkki, $1988 \mathrm{~b})$, D. bicarinata $\left(2 \mathrm{n}=64\left(30 \mathrm{II}+\mathrm{X}_{1} \mathrm{Y}+\mathrm{X}_{2} \mathrm{X}_{3}\right)\right.$ Virkki, $1988 \mathrm{~b})$, D. nigrita $(2 \mathrm{n}=34(16 \mathrm{II}+\mathrm{X}+\mathrm{x})$ Virkki, 1964 and $2 \mathrm{n}=33\left(15 \mathrm{II}+\mathrm{X}_{1} \mathrm{y}+\mathrm{x}_{2}\right)$ Virkki, 1988b) as well as Phenrica austriaca which has been variously described as having a

Send correspondence to Mara Cristina de Almeida. Departamento de Biologia Estrutural, Molecular e Genética, Setor de Ciências Biológicas e da Saúde, Universidade Estadual de Ponta Grossa, Av. Carlos Cavalcanti 4748, Uvaranas, 84.030-900 Ponta Grossa, PR, Brazil. E-mail: almeidamara@uol.com.br. karyotype of $2 \mathrm{n}=49(22 \mathrm{II}+\mathrm{Xy}+3 \mathrm{y})$ by Virkki (1970) and $2 \mathrm{n}=49\left(22 \mathrm{II}+\mathrm{X}_{1} \mathrm{y}+\mathrm{X}_{2}+\mathrm{X}_{3}+\mathrm{X}_{4}\right)$ by Virkki (1988b). In this group, the chromosomal morphology is predominantly metacentric and the sex determination system is variable, with frequent occurrence of multiple systems, involving mainly the X chromosome (Smith and Virkki, 1978; Vidal, 1984; Virkki, 1988b).

In contrast, 74 of the 113 species of the Oedionychina subtribe which have been cytogenetically analyzed have shown high karyotype uniformity concerning the diploid number and type of sex determination system, which is $2 \mathrm{n}=22(20+\mathrm{X}+\mathrm{y})$ with the sex chromosomes being asynaptic during meiosis (Smith and Virkki, 1978; Virkki, 1970, 1971, 1988a, 1989). In the Oedionychina, diploid numbers higher than $2 n=22$ are due to an increase in the number of $y$ chromosome, while numbers lower than $2 n=22$ are due to alterations involving autosomes. In spite of the great number of Oedionychina species studied, little information 
exists on their chromosomal morphology, making it impossible to establish a morphological pattern for the subtribe. Of the 18 Oedionychina species whose chromosomes have been morphologically classified, about $50 \%$ have exhibited solely metacentric autosomes, $25 \%$ only acrocentric autosomes and the other $25 \%$ metacentric and acrocentric autosomes with a predominance of metacentrics (Smith and Virkki, 1978; Virkki, 1985, 1989; Virkki et al. 1991; Virkki and Santiago-Blay, 1993, 1996). In all these species, the X chromosome is invariably metacentric and the y chromosome is predominantly metacentric, with the exception of two species in which the y chromosome is acrocentric.

A peculiarity of all Oedionychini species is the presence of extremely large sex chromosomes, sometimes corresponding to $50 \%$ of the entire genome (Virkki, 1985).

In general, the chromosomes of the Oedionychini species have been analyzed by means of standard staining, and only 7 species have been studied with regard to their C-banding pattern and nucleolus organizer regions (NORs). In these species, the constitutive heterochromatin occurs in the pericentromeric region of all chromosomes, and additionally in the interstitial regions of the sex chromosomes. The use of silver staining to reveal the location of NORs on the chromosomes of these species have shown similar patterns to those produced by C-banding (Virkki, 1983; Virkki and Denton, 1987), which probably does not correspond to the NORs pattern. Consequently, the NORs pattern has not yet been established for this group.

Considering that little karyotype information exists in the literature on Oedionychina species (1 Disonychina and 38 Oedionychina) from the Brazilian fauna, the purpose of this work was to characterize for the first time the karyotype of Paranaita opima in relation to its diploid number, chromosomal morphology, type of sex determination system, constitutive heterochromatin distribution pattern, and NOR-bearing chromosomes.

\section{Material and Methods}

We analyzed 17 Paranaita opima (Germar, 1824) specimens (Figure 1), of which 5 were male embryos and 12 were adults ( 9 males and 3 females). The adults were collected at the Experimental Garden of the Instituto de Biociências, Universidade Estadual Paulista (UNESP), Rio Claro (22 $24^{\prime}$ S, $47^{\circ} 33^{\prime}$ W), São Paulo, Brazil and the embryos were obtained in the laboratory from females naturally fertilized in the field and in captivity.

The mitotic chromosomes were obtained from the embryos and also from the gonads of adult $P$. opima. The embryos and adult gonads were removed in physiological saline solution for insects and were processed according to the methodology of Webb et al. (1978). The testes, but not the embryos, were submitted to a hypotonic treatment (tap water) for $3 \mathrm{~min}$ and then testes and embryos were fixed in Carnoy I mixture (3:1 methanol: acetic acid) for $30 \mathrm{~min}$ and then transferred to a drop of $45 \%(\mathrm{w} / \mathrm{v})$ aqueous acetic acid
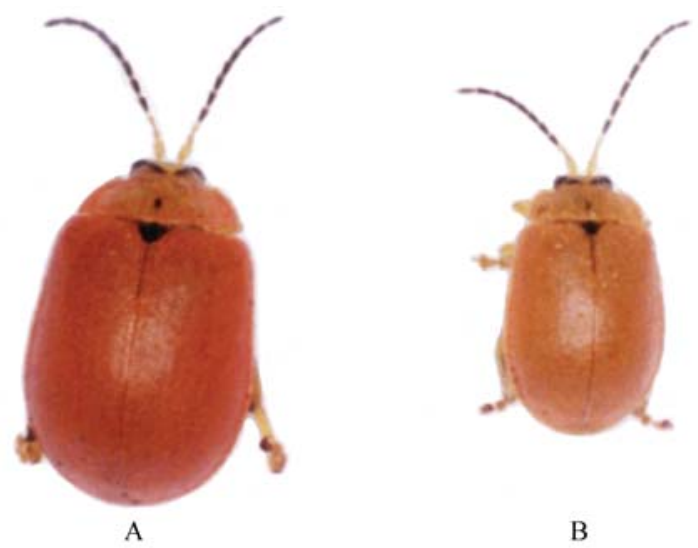

Figure 1 - Specimens of Paranaita opima. A. Female. B. Male. Magnification $=5.2 \mathrm{X}$.

on a microscope slide and the material macerated to form a cell suspension, the slides being dried at $35-40{ }^{\circ} \mathrm{C}$ on a hot-plate, stained with $3 \%(\mathrm{w} / \mathrm{v})$ Giemsa in phosphate buffer ( $\mathrm{pH}$ 6.8) for $12 \mathrm{~min}$, rinsed in distilled water and air-dried. The slides were examined by bright-field optical microscopy using a 100x oil-immersion objective fitted to a Zeiss optical photomicroscope and Kodak Imagelink HQ Microfilm.

The C-banding (Sumner, 1972) and silver nitrate nucleolar organizer region (Ag-NOR) staining (Howell and Black, 1980) were carried out on the Giemsa-stained chromosome preparations described above after removing the immersion oil with Xylene. Triple fluorescent-staining was applied using the GC-specific fluorochrome chromomycin $\mathrm{A}_{3}\left(\mathrm{CMA}_{3}\right)$ and the AT-specific fluorochrome 4'-6-diamidino-2-phenylindole (DAPI), both combined with the distamycin A (DA) counterstain using the technique described by Schweizer (1980).

Routine cytological analyses were carried out as described above and fluorochrome analyses were performed using an Olympus BX50 photomicroscope fitted with filters specific for the DAPI and $\mathrm{CMA}_{3}$ fluorochromes, photomicrographs being made using Kodak T-Max Film. Chromosome morphology was characterized as described by Levan et al. (1964).

\section{Results}

\section{Standard staining with Giemsa}

The $P$. opima mitotic metaphases showed a chromosome complement of $2 \mathrm{n}=22(20+\mathrm{XY})$ for males and $2 n=22(20+X X)$ in females. The karyotype obtained from embryos and gonial metaphases revealed that the diploid complement consists of three submetacentric chromosomes (pairs 1,2 and 5), seven metacentric autosomes (pairs 3, 4 and 6 to 10) and extremely large metacentric $\mathrm{X}$ and $\mathrm{Y}$ sex chromosomes (Figure 2). 


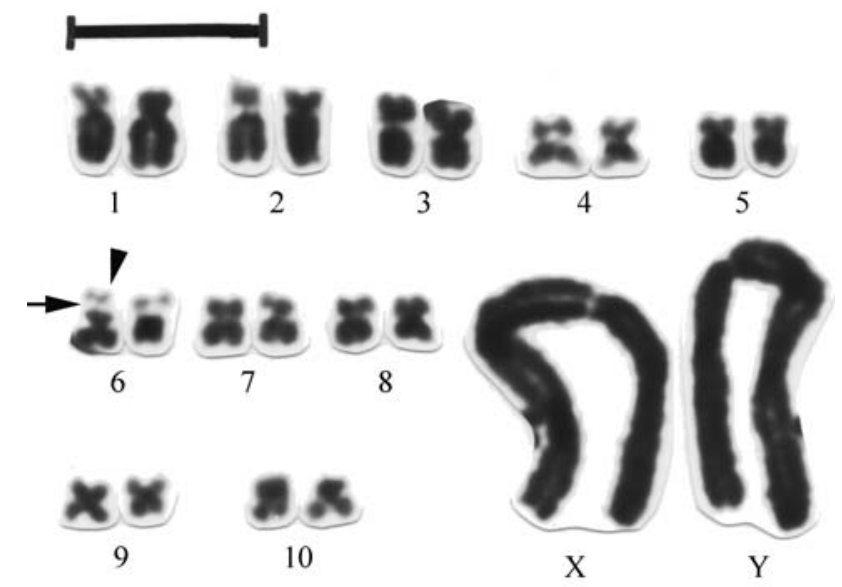

Figure 2 - Giemsa-stained Paranaita opima male embryo with $2 \mathrm{n}=22$ $(20+\mathrm{XY})$ karyotype. Note the secondary constriction (arrow) and the negative heteropycnosis (arrowhead) on pair 6 and the extremely large sex chromosomes. Bar $=10 \mu \mathrm{m}$.

In most of the embryonic, spermatogonial, and oogonial mitotic metaphases, the pericentromeric region of all the chromosomes was negative heteropycnotic. Additionally, in these mitotic metaphases, a prominent secondary constriction was observed at the interstitial region of the short-arm of pair 6 , and the short-arm terminal region of this pair also showed negatively heteropycnotic (Figures 2, $3 \mathrm{~A}, 4 \mathrm{~A}, 6 \mathrm{~A}$ and $6 \mathrm{~F})$.

\section{C-banding}

In mitotic metaphases, all the chromosomes, including the sex chromosomes, showed the occurrence of strongly labeled constitutive heterochromatin in the centromeric region (Figure 3B). In pair 6, the centromeric constitutive heterochromatin extended to the short arm until the secondary constriction (Figures 3B, 6B and 6G).

In some embryonic metaphases, the chromosomes of which were more distended, the Y chromosome showed additional constitutive heterochromatin on the interstitial region of one of the chromosome arms (Figure 3B). In addition, other differential, but not well-defined, marks were visible on the arms of the sex chromosomes.

\section{Ag-NOR staining}

Some Giemsa-stained gonial metaphase cells submitted to Ag-NOR staining showed the NORs at the secondary constriction region of pair 6 (Figures $4 \mathrm{~B}, 6 \mathrm{C}$ and $6 \mathrm{H}$ ).

\section{Triple fluorochrome staining}

Most embryonic mitotic metaphases showed chromosomes with $\mathrm{CMA}_{3}$-negative centromeric regions and homogeneously stained arms (Figure 5A), the exception being pair 6 which showed a $\mathrm{CMA}_{3}$-positive chromosomal region on the short arm which extended from the proximal to the interstitial region (Figure 5A) and was partially coincident with the secondary constriction and the C-band (Fig-

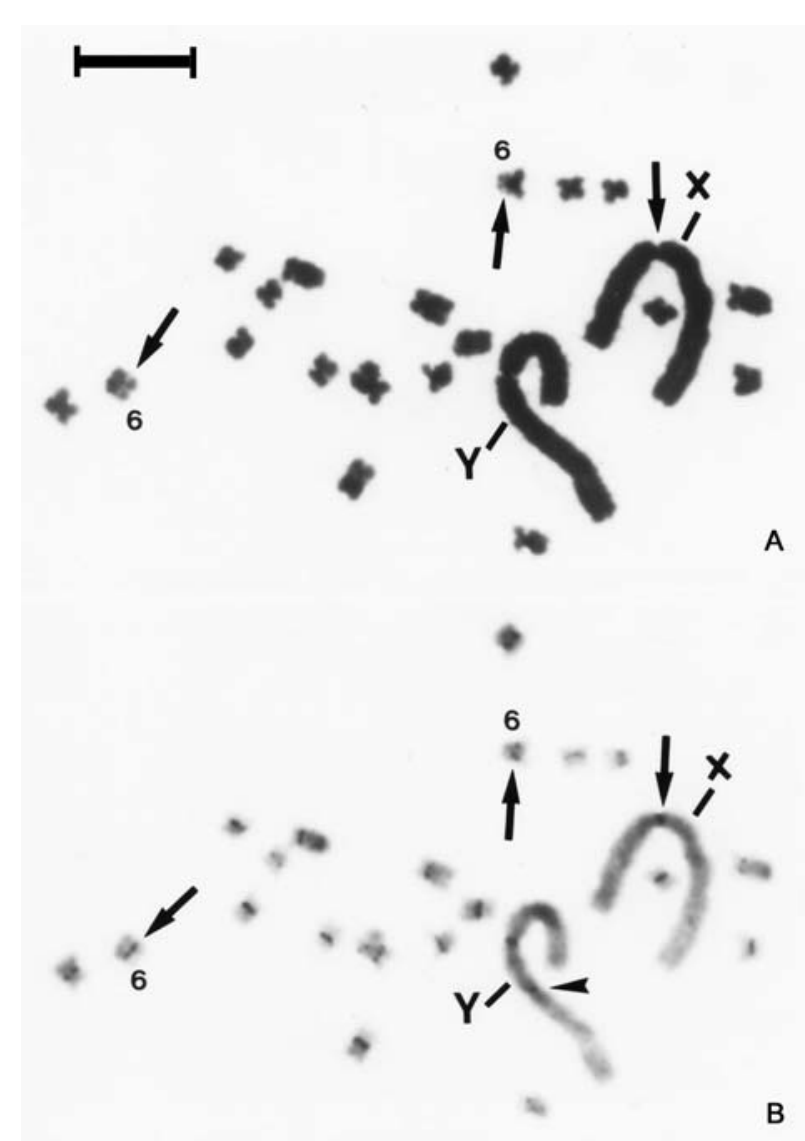

Figure 3 - Male Paranaita opima embryo mitotic metaphase with $2 \mathrm{n}=22$ $(20+\mathrm{XY})$. A. Standard Giemsa staining. B. The same cell as shown in A submitted to Giemsa staining followed by C-banding and showing centromeric constitutive heterochromatin blocks on all chromosomes. Arrows indicate the centromeric region of some chromosomes. The arrowhead points to one of the interstitial C-bands on the sex chromosome. $\mathrm{Bar}=10 \mu \mathrm{m}$

ures $6 \mathrm{~A}, 6 \mathrm{~B}, 6 \mathrm{D}, 6 \mathrm{~F}, 6 \mathrm{G}$ and $6 \mathrm{I})$. Analysis with DAPI filter revealed the presence of fluorescent $\mathrm{AT}$ bands at the centromeric region of all chromosomes of the complement, including the sex chromosomes (Figure 5B). Pair 6 showed DAPI-positive fluorescence at the terminal region of the short arm (Figures 5B, 6E and 6J) in addition to that on the centromeric region and a DAPI-negative region inserted between the centromeric and telomeric DAPI-positive regions, including all positive $\mathrm{CMA}_{3}$ region.

\section{Discussion}

The basic karyotype characteristics of $P$. opima, such as diploid number, chromosomal morphology, type of sex determination system, and C-banding pattern are similar to those described for other Oedionychina species (Virkki, 1961, 1964, 1970; Smith and Virkki, 1978).

The $P$. opima chromosomal number of $2 \mathrm{n}=22$ could have been derived from $2 \mathrm{n}=24$, which has been proposed as ancestral for Chrysomelidae by Virkki (1970) and Smith and Virkki (1978), and may have resulted from fusion 

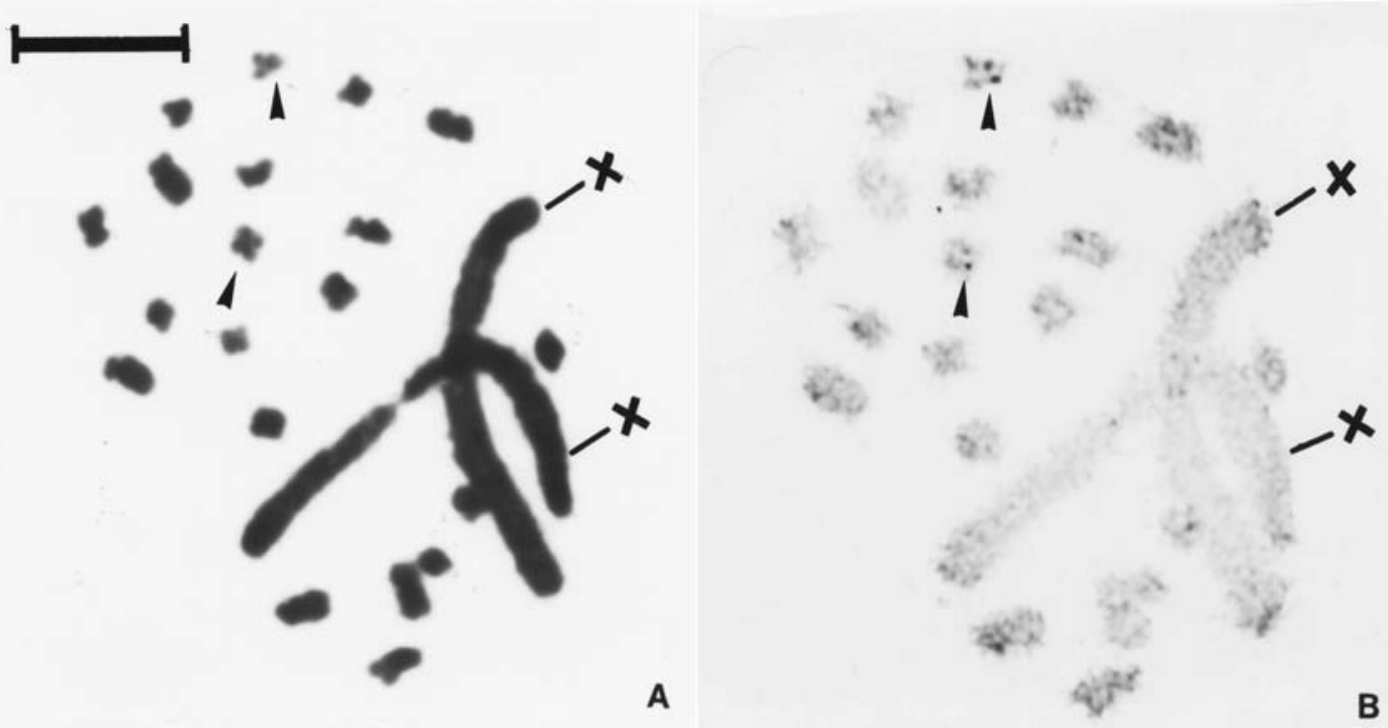

Figure 4 - Adult Paranaita opima oogonial metaphase with $2 n=22(20+X X)$. A. Giemsa staining, showing chromosomal elements of pair 6 (arrowhead). B. The same cell as shown in A, with Giemsa and Ag-NOR sequential staining showing NORs on pair 6 (arrowhead). Bar $=10 \mu \mathrm{m}$.
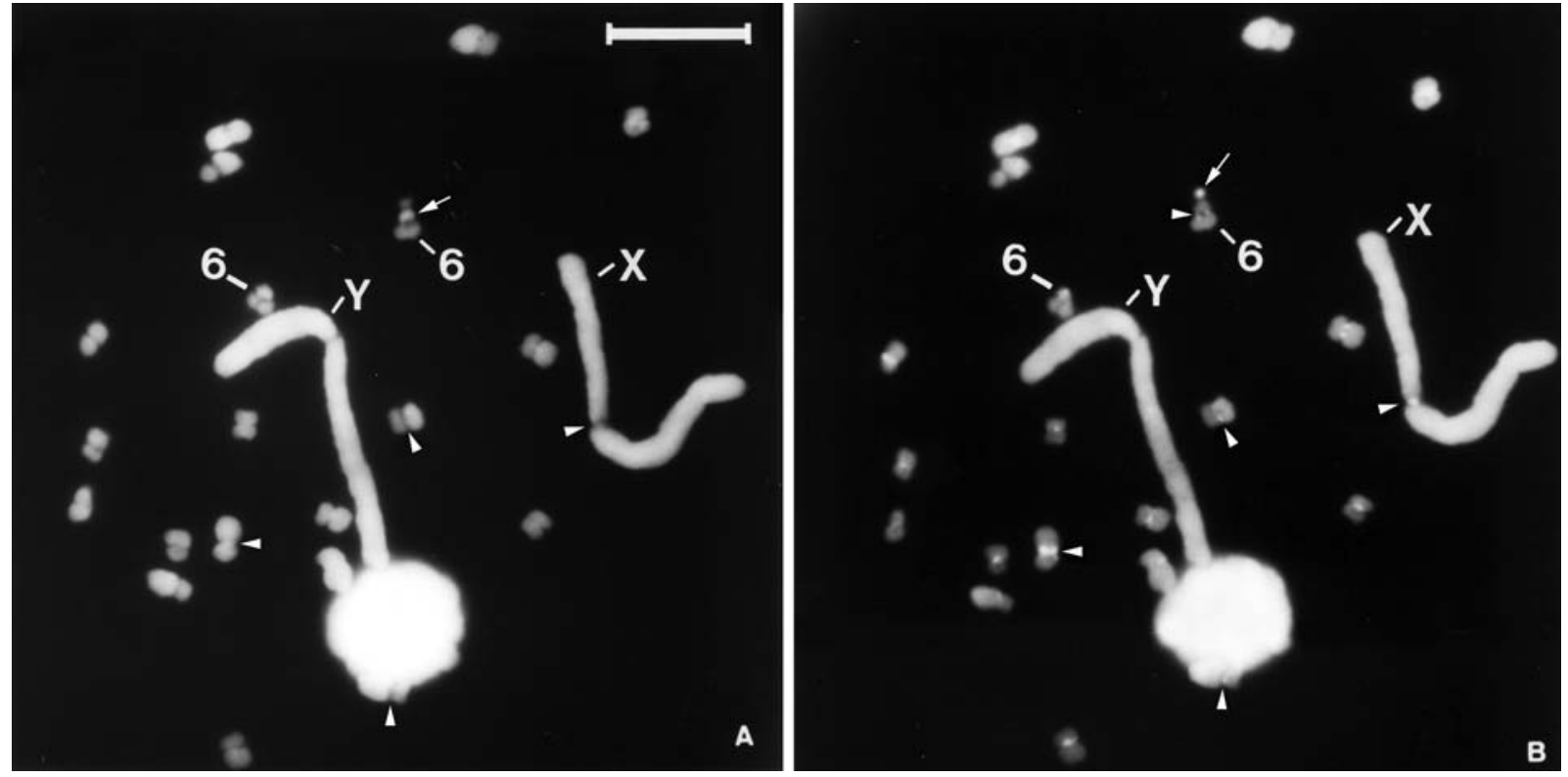

Figure 5 - Male Paranaita opima embryo mitotic metaphase stained with $\mathrm{CMA}_{3}$, DA and DAPI and showing $2 \mathrm{n}=22(20+\mathrm{XY})$. A. Chromomycin $\mathrm{A}_{3}$ staining, showing the $\mathrm{CMA}_{3}$-negative centromeric region (arrowhead) of some chromosomes and the positive $\mathrm{CMA}_{3}$ labeling at the interstitial region of the short arm of pair 6 (arrow). B. DAPI staining showing the DAPI-positive centromeric region (arrowhead) of some chromosomes, the negative DAPI interstitial region of the short arm of pair 6 and the DAPI-positive terminal region of the short arm of pair 6 (arrow). Bar $=10 \mu \mathrm{m}$.

events involving only autosomes or autosomes and sex chromosomes. Similar proposals have been made by Virkki (1970) and Smith and Virkki (1978) for the majority of the Oedionychina species that possesses $2 \mathrm{n}=22$ chromosomes. The presence of metacentric chromosomes in $P$. opima is in agreement with the condition that prevails in the most current Coleoptera species and that is considered by Smith and Virkki (1978) as a basic and ancestral karyotypic characteristic.
Our results show that $P$. opima has similar-sized $\mathrm{X}$ and $\mathrm{Y}$ sex chromosomes, a characteristic that has also been found in two other Oedionychina species (Alagoasa (under Oedionychus) acutangula and Alagoasa extrema), whereas the $\mathrm{X}$ and $\mathrm{y}$ chromosomes of the majority of the species of this subtribe, including Paranaita bilimbata, are different in size. In general, Oedionychina $\mathrm{X}$ and y chromosomes are asynaptic during meiosis but present regular segregation (Virkki, 1968). 


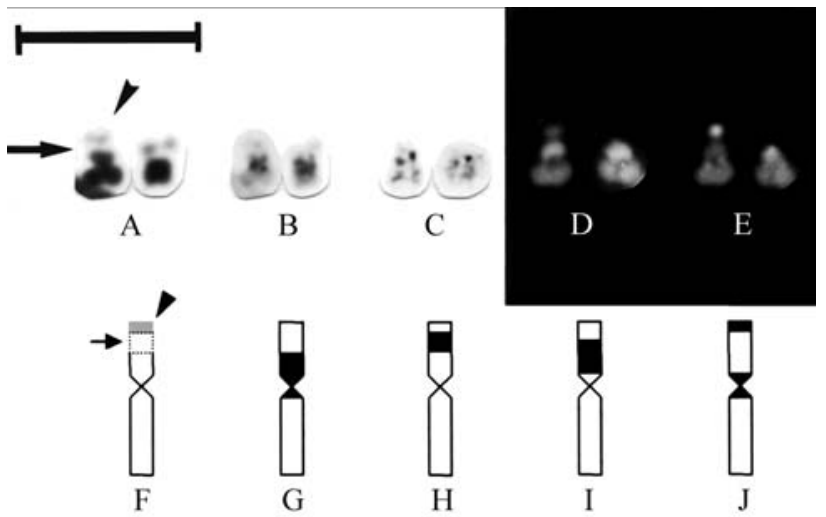

Figure 6 - Paranaita opima pair 6 chromosomal elements submitted to different staining techniques (A-E) and their respective schematic representation (F-J). A and F. Giemsa staining showing secondary constriction (arrow) and negative heteropycnosis (arrowhead) at the short arm terminal region. B and G. C-banding showing a large constitutive heterochromatic block at the pericentromeric region. $\mathrm{C}$ and $\mathrm{H}$. Ag-NORs at the secondary constriction. D and I. $\mathrm{CMA}_{3}$ and DA staining showing the GC-rich DNA at the short arm interstitial region. E and J. DA and DAPI staining showing the AT-rich DNA at the centromere and short arm terminal region.

The C-banding pattern of P. opima was coincident with those described by Virkki (1983) for some Oedionychina species (Omophoita annularis, Omphoita personata, Omophoita octoguttata and Alagoasa januaria) and the P. opima sex chromosomes also exhibit additional C-bands on the interstitial region of the chromosome arms, varying in number, position and intensity according to the degree of chromosome distention, these bands being similar to those described in $O$. annularis, $O$. personata, $O$. octoguttata and A. januaria.

The presence of interstitial C-bands on the large sex chromosomes of Oedionychina species could be related to the origin of this sex determination system in that these bands could represent heterochromatic material remaining from ancient autosomes incorporated into the original sex determination system of the XY type, or from ancestral y chromosomes belonging to the Xny-type sex determination system. According to Virkki (1970), the large and asynaptic sex chromosomes in Oedionychina could be derived from translocations of the $\mathrm{X}$ and $\mathrm{Y}$ chromosomes to a large pair of autosomes, such as those found in Phyllotrupes (Alticinae, Systenini), or from fusions among y chromosomes belonging to the Xny-type multiple sex determination system found in many Disonychina species, depending on the evolutionary relationship between the subtribes.

We found that in P. opima the NORs occur on pair 6, remarkably different from the pattern described for $P$. bilimbata and other Oedionychina species $(O$. annularis, $O$. personata, O. octoguttata, A. januaria, Alagoasa bicolor, Omophoita cyanipennis and Omophoita albicollis), which is characterized by multiple marks on the y chromosome (Virkki, 1983; Virkki and Denton, 1987). According to Virkki (1983), this pattern of multiple marks on the y chromosome could correspond to a peculiar type of chromatin and not to NORs. In other insect groups, the use of silver nitrate impregnation to detect NOR-bearing chromosomes has also shown special types of constitutive heterochromatin and other chromosome structures (Rufas et al., 1983; Cella and Ferreira, 1991).

In the family Chrysomelidae, NORs have been established for a few species (Botanochara angulata, Calligrapha polyspila, Chelymorpha variabilis, Chrysolina americana, Chrysolina bankii and Zatrephrina meticulosa) and are located on one autosomal pair, sometimes at the secondary constriction and sometimes not. In some of these species the NOR-bearing autosomal pair has been identified as either the largest pair or pair 5 (Postiglioni et al., 1990, 1991; Postiglioni and Brum-Zorrilla, 1988; Petitpierre, 1996). The distribution of NORs has only been studied in a few Coleoptera species, but in families such as the Curculionidae and Tenebrionidae the NORs occur on autosomes and on sex chromosomes, while in the Cicindelidae and Coccinellidae they occur on autosomes or on sex chromosomes (Drets et al., 1983; Virkki et al., 1991; Galián et al., 1995; Juan et al., 1993; Maffei et al., 2001).

Our results obtained with the $\mathrm{CMA}_{3}$ and DAPI fluorochromes showed that the centromeric region of all $P$. opima chromosomes is AT-DNA-rich. The presence of DNA with highly repeated AT base sequences in the centromeric region has also been found in the chromosomes of some Tenebrionidae (Juan et al., 1991; Plohl et al., 1993).

In P. opima, the secondary constriction region on pair 6 appeared to include a GC-rich portion in addition to the NOR because one portion of this region was $\mathrm{CMA}_{3}$ positive while the other was silver impregnated and, moreover, all this region was negative for both C-banding and DAPI. On the other hand, the short arm positive DAPI terminal region of pair 6 was $\mathrm{CMA}_{3}$-negative, confirming that this region contains AT base pair sequences. Considering all these results, the NORs on pair 6 seems to be flanked by a special type of heterochromatin, rich in AT base sequences at the terminal side and rich in $\mathrm{GC}$ base sequences at the proximal side. These special types of heterochromatin flanking the NORs were not shown by the usual C-banding technique. The presence of constitutive heterochromatin flanking or inserting NORs has been found in the chromosomes of several different groups of animals (Cabrero et al., 1986; King et al., 1990; Pendás et al., 1993; Silva et al., 2000; Vicari et al., 2003, 2005). The presence of this NOR-associated heterochromatin can restrict genetic recombination in the adjacent region, avoiding the formation of chiasmata or inducing their formation in other chromosome regions (John and King, 1982, 1985) and also can alter the genetic expression of the NORs (Arnold and Shaw, 1985) or can represent breakpoints that facilitate the dispersion of the NORs (Moreira-Filho et al., 1984; Reed Phillips, 1995).

Our results offer important insights into the karyotype characteristics of $P$. opima, which may be useful in eluci- 
dating relationships between species of the subtribe Oedionychina.

\section{Acknowledgments}

The authors thank Dr. Orlando Moreira Filho, from Universidade Federal de São Carlos (UFSCar), Departamento de Genética e Evolução, São Carlos-SP, Brazil for providing facilities in his laboratory to carry out fluorochrome staining and analysis of the material. We also thank Dr. Sanae Kasahara, from Universidade Estadual Paulista (UNESP), Departamento de Biologia, Rio Claro-SP, Brazil for her suggestions and critical reading of the manuscript. This work was supported by two Brazilian Agencies, Coordenação de Aperfeiçoamento de Pessoal de Nível Superior (CAPES) and Fundação Araucária-PR.

\section{References}

Arnold ML and Shaw DD (1985) The heterochromatin of grasshoppers from the Caledia captiva species complex. II. Cytological organisation of tandemly repeated DNA sequences. Chromosoma 93:183-190.

Cabrero J, Navas-Castillo J and Camacho JPM (1986) Effects of supernumerary chromosome segments on the activity of nucleolar organiser regions in the Chorthipus binotatus. Chromosoma 93:375-380.

Cella DM and Ferreira A (1991) The cytogenetics of Abracris flavolineata (Orthoptera, Caelifera, Ommatolampinae, Abracrini). Rev Bras Genet 14:315-329.

Drets ME, Corbella E and Folle GA (1983) C-banding and nonhomologous associations. II. The "parachute" Xyp sex bivalent and the behavior of heterochromatic segments in Epilachna paenulata. Chromosoma 88:249-255.

Galián J, Serrano J, De La Rúa P, Petitpierre E and Juan C (1995) Localization and activity of rDNA genes in tiger beetles (Coleoptera, Cicindelidae). Heredity 74:524-530.

Howell WM and Black DA (1980) Controlled silver staining of nucleolus organizer regions with protective colloidal developer: A 1-step method. Experientia 36:1014-1015.

John B and King M (1982) Meiotic effects of supernumerary heterochromatin in Heteropternis obscurella. Chromosoma 85:39-65.

John B and King M (1985) The inter-relationship between heterochromatin distribution and chiasma distribution. Genetica 66:183-194.

Juan C, Gosálvez J, Mezzanotte R and Petitpierre E (1991) Cytological and biochemical characterization of the in situ endonuclease digestion of fixed Tenebrio molitor chromosomes. Chromosoma 100:432-438.

Juan C, Pons J and Petitpierre E (1993) Localization of tandemly repeated DNA sequences in beetle chromosomes by fluorescent in situ hybridization. Chromosome Res 1:167-174.

King M, Contreras N and Honeycut RL (1990) Variation within and between nucleolar organizer regions in Australian hylid frogs (Anura) shown by $18 \mathrm{~s}+28 \mathrm{~s}$ in situ hibridization. Genetica 80:17-29.

Levan A, Fredga K and Sandberg AA (1964) Nomenclature for centromeric position on chromosomes. Hereditas 52:201220 .
Maffei EMD, Fragoso DB, Pompolo SG and Serrão JE (2001) Morphological and cytogenetical studies on the female and male reproductive organs of Eriops connexa Mulsant (Coleoptera, Polyphaga, Coccinellidae). Neth J Zool 51:483-496.

Moreira-Filho O, Bertollo LAC and Galetti PMJr (1984) Structure and variability of nucleolar organizer regions in Parodontidae fish. Can J Genet and Cytology 26:564-568.

Pendás AM, Morán P and Garcia-Vázquez E (1993) Ribosomal RNA genes are interspersed throughout a heterochromatic chromosome arm in Atlantic Salmon. Cytogenet Cell Genet 63:128-130.

Petitpierre E (1996) Molecular cytogenetics and taxonomy of insects, with particular reference to the Coleoptera. Int J Insect Morphol Embryol 25:115-134.

Plohl M, Lucijanic-Justic V, Ugarkovic D, Petitpierre E and Juan C (1993) Satellite DNA and heterochromatin of the flour beetle Tribolium confusum. Genome 36:467-475.

Postiglioni A and Brum-Zorrilla N (1988) Non-relationship between nucleolus and sex chromosome system Xyp in Chelymorpha variabilis Boheman (Coleoptera, Chrysomelidae). Genetica 77:137-141.

Postiglioni A, Mazzella MC, Panzera F, Silva A da, León RP de, Kvasina L and Scvortzof F (1990) Sex chromosomes of neotropical Coleoptera from Uruguay. Nucleus 33:25-30.

Postiglioni A, Stoll M and Brum-Zorrilla N (1991) Haploid karyotype analysis of Chelymorpha variabilis Bohemam (Coleoptera, Chrysomelidae) with microspreading techniques. Rev Bras Genet 14:653-660.

Reed KM and Phillips RB (1995) Molecular cytogenetic analysis of the double- $\mathrm{CMA}_{3}$ chromosome of lake trout, Salvelinus namaycush. Cytogenet Cell Genet 70:104-107.

Rufas JS, Gosálves J, Gimenéz-Martín G and Esponda P (1983) Localization and development of kinetochores and a chromatid core during meiosis in grasshopper. Genetica 61:233238.

Schweizer D (1980) Simultaneous fluorescent staining of R bands and specific heterochromatin regions (DA-DAPI bands) in human chromosomes. Cytogenet Cell Genet 27:190-193.

Silva APZ, Baldissera Jr FA, Haddad CFB and Kasahara S (2000) Karyotypes and nucleolus organizer regions in four species of the genus Physalaemus (Anura, Leptodactylidae). Iheringia Zool 88:159-164.

Smith SG and Virkki N (1978) Animal Cytogenetics, v. 3. Insecta 5. Coleoptera. Gebruder Borntraeger, Berlin, $366 \mathrm{pp}$.

Sumner AT (1972) A simple technique for demonstrating centromeric heterochromatin. Exp Cell Res 75:304-306.

Vicari MR, Artoni RF and Bertollo LAC (2003) Heterochromatin polymorphism associated with 18S rDNA: A differential pathway among Hoplias malabaricus fish populations. Cytogenet Genome Res 101:24-28.

Vicari MR, Artoni RF and Bertollo LAC (2005) Comparative cytogenetics of Hoplias malabaricus (Pisces, Erythrinidae): A population analysis in adjacent hydrographic basins. Genet Molec Biol 28:103-110.

Vidal OR (1984) Chromosome numbers of Coleoptera from Argentina. Genetica 65:235-239.

Virkki N (1961) Non-conjugation and late conjugation of the sex chromosomes in the beetles of the genus Alagoasa (Chrysomelidae, Alticinae). Ann Acad Sci Fenn A IV 54:1-22. 
Virkki N (1964) On the cytology of some neotropical chrysomelids (Coleoptera). Ann Acad Sci Fenn A IV 75:1-24.

Virkki N (1968) Regular segregation of seven asynaptic sex chromosomes in the male of Asphaera daniela Bechyné (Coleoptera, Alticinae). Caryologia 21:47-51.

Virkki N (1970) Sex chromosomes and karyotypes of the Alticidae (Coleoptera). Hereditas 64:267-282.

Virkki N (1971) Formation and maintenance of the distance sex bivalent in Oedionychina (Coleoptera, Alticidae). Hereditas 68:305-312.

Virkki N (1983) Banding of Oedionychina (Coleoptera, Alticinae) chromosomes. C- and Ag-bands. J Agriculture Univ P R 67:221-255.

Virkki N (1985) The cytogenetic system of Oedionychina (Alticinae). First Int Symp Chrysom 3:489-497.

Virkki N (1988a) Cytotaxonomy of Alticinae. Jolivet E, Petitpierre E and Hsiao TH (eds) Biology of Chrysomelidae. Kluwer Academic Publishers, Norwell, pp 187-203.
Virkki N (1988b) The sex chromosomes of Disonychina (Coleoptera, Alticinae): $\mathrm{Xy}+\mathrm{nX}$ systems. Cytobios 53:43-55.

Virkki N (1989) Proximal vs. distal collochores in coleopteran chromosomes. Hereditas 110:101-107.

Virkki N and Denton A (1987) Silver staining of the elements of spermatogenesis in Oedionychina (Chrysomelidae, Alticinae). Hereditas 106:37-49.

Virkki N, Mazzella C and Denton A (1991) Silver staining of the coleopteran Xyp sex bivalent. Cytobios 67:45-63.

Virkki N and Santiago-Blay JA (1993) Trends of karyotype evolution in neotropical Oedionychina (Coleoptera, Chrysomelidae, Alticinae). Hereditas 119:263-283.

Virkki N and Santiago-Blay JA (1996) Chromosomes of some Puerto Rican fleabeetles (Coleoptera, Chrysomelidae, Alticinae): Multiple cytogenetic evolutionary tendencies in neotropics. Journal Zoological Evolution Research 34:113-119.

Webb GC, White MJD, Contreras N and Cheney J (1978) Cytogenetics of the parthenogenetic grasshopper Warramaba (formely Moraba) virgo and its bisexual relatives. IV. Chromosomes banding studies. Chromosoma 67:309-339. Associate Editor: Yatiyo Yonenaga-Yassuda 